\title{
The Association of Sleep Duration and the Use of Electronic Cigarettes, NHANES, 2015-2016
}

\author{
R. Constance Wiener $\left(\mathbb{D},{ }^{1}\right.$ Christopher Waters $\mathbb{D}^{2},{ }^{2}$ Ruchi Bhandari $\mathbb{D}^{3},^{3}$ \\ Alcinda K. Trickett Shockey ${ }^{1}{ }^{4}{ }^{4}$ and Omayma Alshaarawy ${ }^{5}$ \\ ${ }^{1}$ West Virginia University, Department of Dental Practice and Rural Health, School of Dentistry, 104a Health Sciences Addition, \\ PO Box 9415, Morgantown, WV 26506, USA \\ ${ }^{2}$ West Virginia University, Department of Dental Research, School of Dentistry, 106a Health Sciences Addition, PO Box 9448, \\ Morgantown, WV 26506, USA \\ ${ }^{3}$ West Virginia University, Department of Epidemiology, School of Public Health, Robert C Byrd Health Sciences Center North, \\ Room G104C, Morgantown, WV 26506, USA \\ ${ }^{4}$ West Virginia University, Department of Dental Hygiene, School of Dentistry, Robert C Byrd Health Sciences Center North, \\ Room 1192A, Morgantown, WV 26506, USA \\ ${ }^{5}$ Michigan State University, Department of Family Medicine, College of Human Medicine, Room B113, 788 Service Road, Lansing, \\ Michigan 48824, USA
}

Correspondence should be addressed to Ruchi Bhandari; rbhandari@hsc.wvu.edu

Received 29 August 2019; Revised 7 January 2020; Accepted 19 February 2020; Published 3 March 2020

Academic Editor: Luigi Ferini-Strambi

Copyright (C) 2020 R. Constance Wiener et al. This is an open access article distributed under the Creative Commons Attribution License, which permits unrestricted use, distribution, and reproduction in any medium, provided the original work is properly cited.

\begin{abstract}
Introduction. The unknown effects of electronic cigarettes are public health concerns. One potential effect of electronic cigarette fluid constituents, such as nicotine, may influence sleep. The purpose of this study is to determine if there is an association between sleep duration and electronic cigarette use. Methods. A retrospective, cross-sectional study was conducted using National Health and Nutrition Examination Survey (NHANES) 2015-2016. Variables of interest included responses to questions concerning electronic cigarette use, hours of sleep, and other variables associated with sleep. Data analyses were conducted with the Rao-Scott chi square test and logistic regression. Results. This study was conducted on 2889 participants, aged 18-65 years, of whom $50.7 \%$ were female. Using a bivariate analyses of electronic cigarette usage and sleep duration, participants who never used an electronic cigarette were more likely to have appropriate sleep durations as compared with participants who were currently using electronic cigarettes $(P<0.0001)$. After adjusting for sociodemographic variables and cigarette smoking, current electronic cigarette use was associated with higher odds of less sleep duration (adjusted odds ratio $=1.82 ; 95 \%$ CI: 1.18, 2.79; $P=0.0075)$. Conclusions. Participants currently using electronic cigarettes are more likely to have less sleep as compared to participants who have never used electronic cigarettes. Implications. With sleep time duration being a major factor for proper body function and repair, this study can serve as confirmation that the use of electronic cigarettes is not a harmless health behavior.
\end{abstract}

\section{Introduction}

Inadequate sleep duration is a public health concern. It is associated with higher all-cause mortality with poor sleep duration [1], higher cancer mortality [2], and increased rates of cardiovascular diseases, diabetes, and obesity [3]. Researchers have related smoking and tobacco use disorder to several sleep disturbances, including poor sleep efficiency, sleep onset latency, number of awakenings [4-6], sleep bruxism, restless leg syndrome [7], and sleep-disordered breathing [8, 9].

The association of sleep and smoking is not fully understood. A nicotine pathway has been postulated as one potential mechanism. It is theorized that the nicotine in tobacco products activates the sympathoadrenal system thereby increasing heart rate, blood pressure, and glucose levels [10]. The activation has a potential to delay or interfere with sleep. 
Researchers showed such an effect in preclinical studies where nicotine administration reduced total sleep time duration due to its stimulatory actions [11]. Additionally, researchers found polysomnographic indications of longer sleep onset latency, less total sleep time duration, decreased sleep continuity, and increased wake time among current smokers $[9,12]$. Other sleep impairments and insomnia have been noted and associated with the removal of nicotine and its effects during tobacco cessation [12].

Conversely, there are mixed results of sleep disorders and tobacco use in some studies. In a cross-sectional study of young adults, researchers found that sleep quality, sleep time duration, and sleep onset latency were similar among smokers and nonsmokers [13]. In another study, there was no significant correlation of smoking status with apnea hypopnea index, sleep onset latency, and several other measures of sleep quality [14]. When the sample was stratified by age, total sleep time and sleep efficiency were higher and statistically significant in the smoker/former smoker group.

There are few similar studies in which researchers have examined electronic cigarette use and sleep duration. Electronic cigarette devices create an aerosol from a fluid (e-liquid). The e-liquid components can vary immensely. Commercial e-liquids often consist of vegetable glycerin, propylene glycol, proprietary flavorings, and nicotine [15]. The liquids are heated resulting in the formation of an aerosol and are directly inhaled [15]. There were 466 brands and 7764 different flavors of electronic cigarettes identified online from May-August 2012 to December 2013-January 2014 [16]. Many electronic cigarette e-liquids have not been tested. E-liquid fruit flavors, for example, typically contain diacetyl, which has been linked to bronchiolitis obliterans, an irreversible respiratory disease [17]. Some strawberry flavored e-liquids were found to be cytotoxic [17]. There is a concern that vitamins in some e-liquids may cause pulmonary damage [17].

The use of electronic cigarettes has been increasing. Nearly one-fifth (21.6\%) of adults, aged 18-24 years, tried electronic cigarettes in 2014 [18]. Approximately 59\% of individuals were dual users, reporting both electronic cigarette use and cigarette use [19]. Among high school students, electronic cigarette use increased from $11.7 \%$ in 2007 to $20.8 \%$ in 2018 [20]. It is important to understand the effect of these products on health.

Currently, there are very few studies on sleep duration and electronic cigarettes, and of the studies, controversy remains as to if an association exists. Researchers recently concluded that dual use of electronic cigarettes with conventional tobacco is associated with decreased sleep quality in women [21]. Other researchers who examined sleep data on 2488 California adolescents found no significant difference in weekday sleep time duration between adolescents who used electronic cigarettes at a higher frequency and lower frequency, although adolescents who used electronic cigarettes slept significantly less on weekends compared with adolescents who did not use electronic cigarettes [22].

It is known that many factors affect sleep duration. In the Unifying Energy Allocation Model of Sleep (the theoretical basis for this study), there are functions that generally occur in wakefulness (including, vigilance, foraging, and reproduction) that are downregulated during sleep; and there are functions that generally occur during sleep (including growth, cellular housekeeping, repair, immune function, and neural network reorganization) and are downregulated during wakefulness [23]. The predominant functions and wake/sleep cycle are altered by drugs/products, alcohol, infection, activity, anxiety/stress, chronic disease, and other factors. In this study, we examined if electronic cigarettes are among the drugs/products that alter sleep duration. However, as this study has a cross-sectional study design, causality cannot be determined. Therefore, questions about the association of electronic cigarette use and sleep remain unanswered. The purpose of this study is to determine the association of electronic cigarette use and sleep time duration among adults covering a wide range of ages (18-65 years). Data are retrieved from the National Health and Nutrition Examination Survey (NHANES) 2015-2016.

\section{Methods}

This study received West Virginia University Institutional Review Board acknowledgement of nonhuman subject research (Protocol number 1907635442). Data used in this study were accessed from the NHANES, 2015-2016, available online at https://wwwn.cdc.gov/nchs/nhanes/ ContinuousNhanes/Default.aspx?BeginYear=2015 [24]. The NHANES is a program which began in the 1960 s to determine population health based upon approximately 5000 noninstitutionalized U.S. residents. NHANES participants respond to questions concerning demographics, diet, socioeconomic status, and health status. They also undergo dental and medical examinations, laboratory tests, and physiological measurement. The NHANES uses a complex study design to allow for the generalization of findings. Weights and sampling adjustments are provided in the data sets.

The data used in this study were from the Questionnaire Data files of NHANES. The question used for the sleep variable was "How much sleep do you usually get at night on weekdays or workdays?" [24]. The participants' sleep duration responses, in hours, were categorized into "not recommended for their age" and "may be appropriate for their age," based on the National Sleep Foundation sleep times. For individuals aged 18-25 years, a not recommended sleep duration is $<6$ hours and $>11$ hours; for individuals aged 26-64 years, a not recommended sleep duration is $<6$ hours and $>10$ hours; and for individuals $\geq 65$ years, a not recommended sleep duration is $<6$ hours and $>9$ hours [25]. Therefore, "may be appropriate" sleep durations in this study were 6 to 11 hours for individuals aged 18-25 years, 6 to 10 for individuals aged 26-64 years, and 5-9 hours for individuals $\geq 65$ years. The National Sleep Foundation indicates 6 hours to 10 or 11 hours for individuals aged 18-25 years and 5 or 6 hours to 9 hours for individuals aged $\geq 65$ years in the "may be appropriate" sleep duration category (which slightly overlaps the "not recommended" category) [25].

In NHANES, electronic cigarette use questions were asked in the participant's residence by calibrated interviewers using the Computer-Assisted Personal Interview system. The 


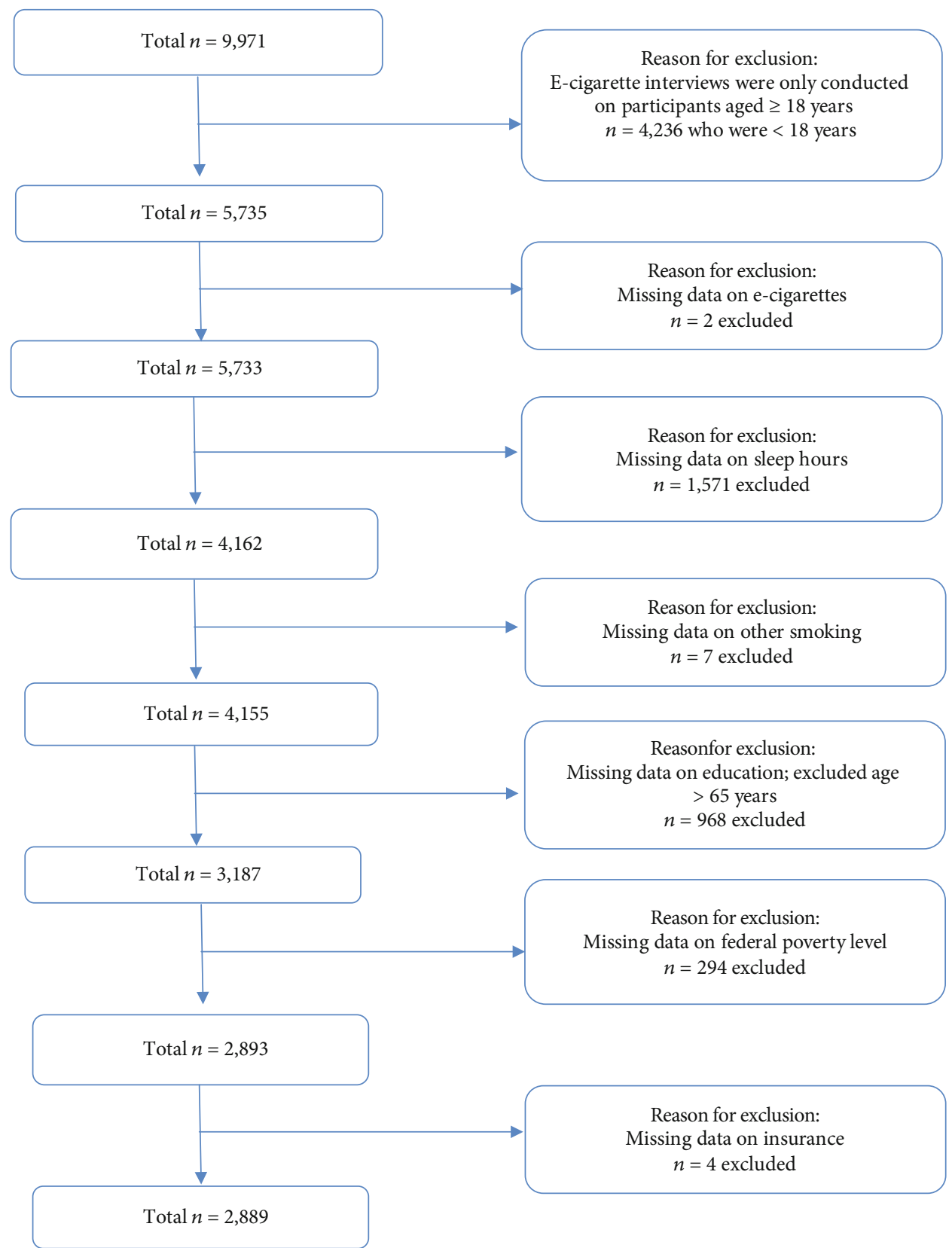

FIGURE 1: Study sample.

questions used for the electronic cigarette variable were "Have you ever used an electronic cigarette even one time?" and "During the past 30 days, on how many days did you use electronic cigarettes?" [24]. A negative response to the first question was used to define the participant as a person who never used electronic cigarettes. A positive response to the first question and a response of not having used an electronic cigarette during the past 30 days were used to define the participant as a person who formerly used electronic cigarettes. A positive response to the first question and a response of having used an electronic cigarette within the previous 30 days were used to define the participant as a person who currently uses electronic cigarettes. The authors recognize the complexity of evaluating electronic cigarette use. Whereas never cigarette smoking is generally recognized as fewer than 100 lifetime cigarettes and current cigarette smoking is currently smoking some or every day, similar definitions are not standard for electronic cigarette use [26-28].

In order to more fully describe the sample, electronic cigarette use was further subgrouped by frequency of use over the past 30 days: (1) daily use, (2) intermediate use for more than 5 days to fewer than 30 days, and (3) infrequent use of 1 day to 5 days. Also, to more fully describe the sample, a dual use category was created: (1) current dual use (use of cigarettes and electronic cigarettes over the previous 30 days); (2) never dual use (any smoking category (current, former, never) and never electronic cigarette use or any electronic cigarette use category (current, former, never) and never 
TABLE 1: Sample characteristics, NHANES 2015-2016, $n=2889$.

\begin{tabular}{|c|c|c|}
\hline & Number & Weighted percentage \\
\hline \multicolumn{3}{|l|}{ Electronic cigarette use $^{1}$} \\
\hline Current & 191 & 7.1 \\
\hline Daily use & 38 & 1.5 \\
\hline Intermediate use & 35 & 1.6 \\
\hline Infrequent use & 118 & 4.0 \\
\hline Former & 457 & 17.9 \\
\hline Never & 2241 & 75.0 \\
\hline \multicolumn{3}{|l|}{ Cigarette use } \\
\hline Current use & 626 & 21.7 \\
\hline Former use & 480 & 19.5 \\
\hline Never use & 1783 & 58.8 \\
\hline \multicolumn{3}{|l|}{ Dual use ${ }^{2}$} \\
\hline Current & 116 & 4.6 \\
\hline Former & 94 & 4.2 \\
\hline Never & 2679 & 91.2 \\
\hline \multicolumn{3}{|l|}{ Sex } \\
\hline Female & 1496 & 50.7 \\
\hline Male & 1393 & 49.3 \\
\hline \multicolumn{3}{|l|}{ Age in years } \\
\hline $18-25$ & 563 & 18.0 \\
\hline $26-44$ & 1140 & 40.0 \\
\hline $45-65$ & 1186 & 42.1 \\
\hline \multicolumn{3}{|l|}{ Race/ethnicity } \\
\hline Non-Hispanic white & 832 & 60.0 \\
\hline Non-Hispanic black & 669 & 12.7 \\
\hline Mexican American & 904 & 17.3 \\
\hline Other & 484 & 10.1 \\
\hline \multicolumn{3}{|l|}{ Education } \\
\hline High school/less & 1383 & 37.6 \\
\hline More than HS & 1506 & 62.4 \\
\hline \multicolumn{3}{|l|}{ Federal poverty level } \\
\hline$\leq 200 \%$ & 1525 & 39.7 \\
\hline$>200 \%$ & 1364 & 60.3 \\
\hline \multicolumn{3}{|l|}{ Health insurance } \\
\hline Yes & 2233 & 82.6 \\
\hline No & 656 & 17.4 \\
\hline \multicolumn{3}{|l|}{ Body mass index } \\
\hline Under/normal & 1149 & 40.0 \\
\hline Over/obese & 707 & 60.0 \\
\hline \multicolumn{3}{|l|}{ Alcohol use } \\
\hline No & 848 & 22.7 \\
\hline Moderate & 870 & 36.5 \\
\hline Heavy & 720 & 27.2 \\
\hline Missing & 451 & 13.6 \\
\hline \multicolumn{3}{|l|}{ Chronic disease $^{2}$} \\
\hline Yes & 919 & 30.4 \\
\hline No & 1970 & 69.6 \\
\hline
\end{tabular}

TABLE 1: Continued.

\begin{tabular}{lcc}
\hline & Number & Weighted percentage \\
\hline Sleepiness & & \\
0-1/month & 1197 & 37.7 \\
2-4/month & 930 & 34.2 \\
$\quad$ 5/month & 762 & 28.1 \\
Snoring & & \\
$\quad$ 0-2/week & 1527 & 58.3 \\
3-4/week & 483 & 16.4 \\
5 or more times/week & 716 & 25.3 \\
Snorting & & \\
0-2/week & 2439 & 89.1 \\
3-4/week & 159 & 5.6 \\
5 or more times/week & 142 & 5.3 \\
Sleep duration for specific age ${ }^{3}$ & & \\
$\quad$ May be appropriate & 2505 & 10.2 \\
$\quad$ Not recommended & 384 & \\
\hline
\end{tabular}

Abbreviations: wt\%: weighted column percent; HS: high school. ${ }^{1}$ Current use $=$ daily use over the past 30 days; intermediate use $=$ use $>5$ days to $\leq 29$ days; infrequent use $=\geq 1$ day to $\leq 5$ days. ${ }^{2}$ Dual use current $=$ smoking cigarettes and using electronic cigarettes over the previous 30 days; former dual use $=$ reporting having smoked $>100$ lifetime cigarettes and having ever used electronic cigarettes; never = reporting never having smoked $>100$ cigarettes and reporting never having tried electronic cigarettes. ${ }^{3}$ Chronic disease $=$ positive response to having arthritis, cardiovascular disease, depression, emphysema, or diabetes. ${ }^{4}$ Based upon National Sleep Foundation (https://www.sleepfoundation.org/press-releasenational-sleepfoundation-recommends-new-sleep-times).

cigarette use); and (3) former dual use (smoked $>100$ lifetime cigarettes but not having used cigarettes over the past 30 days and used electronic cigarettes but not having used them over the past 30 days).

Based on previous research and the Unifying Energy Allocation Model of Sleep, the following variables were associated with both electronic cigarette use and sleep duration and were included in the study: sex (male, female); age (18-25 years, 26-44 years, 45-65 years); race/ethnicity (non-Hispanic white, non-Hispanic black, Mexican American, other); education (high school or less, more than high school); federal poverty level $(\leq 200 \%,>200 \%)$; health insurance (yes, no); cigarette smoking (current, former, never); body mass index (underweight/normal, overweight/obese); alcohol use (no, moderate, heavy, missing); presence of chronic disease defined as arthritis, cardiovascular disease, depression, emphysema, or diabetes (yes, no); and report of daytime sleepiness ( $0-1 /$ month, $2-4 /$ month, $\geq 5 /$ month), a measure of sleep quality (Figure 1 ).

Variables were analyzed for frequency and weighted percentages, presented by current, former, and never electronic cigarette use. Chi-square test was conducted to identify bivariate associations between sleep duration categories (not having recommended sleep duration for their age and may be appropriate sleep duration for their age). The analyses were adjusted for strata, design, and sample weights accounting for the complex sampling design. The key concepts for adequate sample sizes when using NHANES data 
TABLE 2: Bivariate analysis of electronic cigarette use with sample variables, NHANES 2015-2016, $n=2889$.

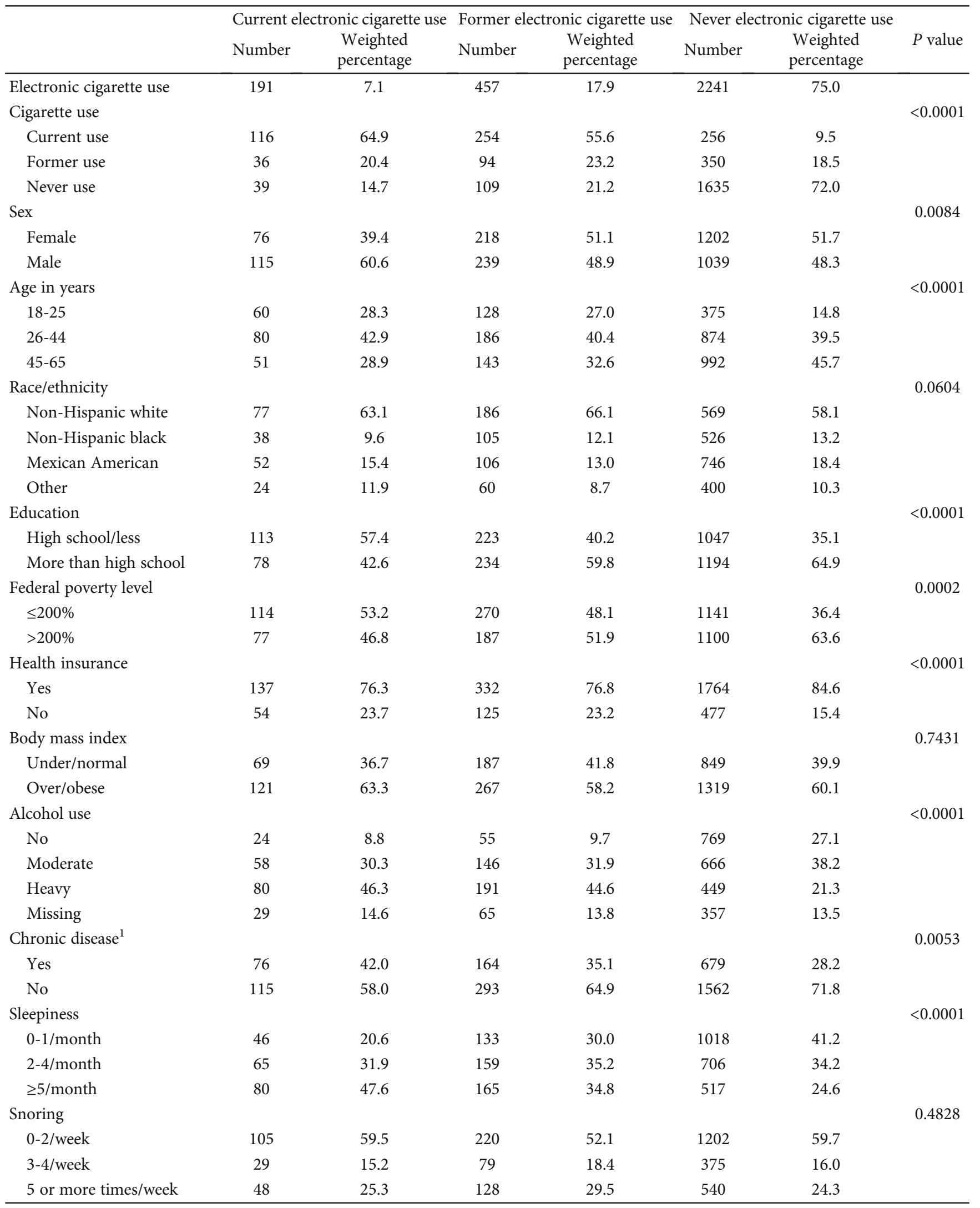


TABLE 2: Continued.

\begin{tabular}{|c|c|c|c|c|c|c|c|}
\hline & \multicolumn{2}{|c|}{ Current electronic cigarette use } & \multicolumn{2}{|c|}{ Former electronic cigarette use } & \multicolumn{2}{|c|}{ Never electronic cigarette use } & \multirow[b]{2}{*}{$P$ value } \\
\hline & & $\begin{array}{l}\text { Weighted } \\
\text { percentage }\end{array}$ & Number & $\begin{array}{l}\text { Weighted } \\
\text { percentage }\end{array}$ & Number & $\begin{array}{l}\text { Weighted } \\
\text { percentage }\end{array}$ & \\
\hline Snorting & & & & & & & 0.0034 \\
\hline 0 -2/week & 151 & 84.2 & 372 & 85.3 & 1916 & 90.5 & \\
\hline 3-4/week & 18 & 11.0 & 24 & 5.7 & 117 & 5.0 & \\
\hline 5 or more times/week & 13 & 4.8 & 39 & 9.0 & 90 & 4.5 & \\
\hline Sleep duration for specific age ${ }^{2}$ & & & & & & & $<0.0001$ \\
\hline Not recommended & 41 & 19.9 & 67 & 9.9 & 276 & 9.4 & \\
\hline May be appropriate & 150 & 80.1 & 390 & 90.1 & 1965 & 90.6 & \\
\hline
\end{tabular}

Abbreviations: wt\%: weighted column percent; HS: high school. $P$ values indicate Rao-Scott chi-square test Pr>ChiSq for current/former/never electronic cigarette use. ${ }^{1}$ Chronic disease $=$ positive response to having arthritis, cardiovascular disease, depression, emphysema, or diabetes. ${ }^{2}$ Based upon National Sleep Foundation (https://www.sleepfoundation.org/press-releasenational-sleep-foundation-recommends-new-sleep-times).

are that the relative standard error should be less than $30 \%$ (standard of the estimate error divided by the value of the estimate) and that the degrees of freedom are calculated by the number of primary sampling units (clusters) minus the number of strata. These criteria limited the number of variables that could be considered in the analyses. Bivariate and multivariable logistic regression analyses were conducted to examine the strength of association of electronic cigarette use and sleep duration. Data analyses were conducted with SAS $^{\circledR}$ version 9.4 (SAS Institute, Inc., Cary, NC). The significance level was set, a priori, at $P<0.05$.

\section{Results}

The sample description is presented in Table 1 . There were 2889 participants, of whom $50.7 \%$ were female. There were $18.0 \%$ who were aged $18-25$ years, $40.0 \%$ who were $26-44$ years, and $42.1 \%$ who were $45-65$ years. Most of the participants were non-Hispanic white $(60.0 \%)$, overweight/obese (60.0\%), had no chronic diseases $(69.6 \%)$, and were $>200 \%$ of the federal poverty level. There were $21.7 \%$ current smokers. Approximately $7.1 \%$ of the sample reported currently using electronic cigarettes. Of those participants, $1.5 \%$ used electronic cigarettes daily, $1.6 \%$ used them intermittently, and $4.0 \%$ used them infrequently. About $4.6 \%$ of participants had dual electronic cigarette and combustible cigarette use.

Bivariate relationships of electronic cigarette use with the variables of interest are presented in Table 2. Significant relationships with electronic cigarettes were with less sleep duration, older age, less education, lower federal poverty level, lack of health insurance, concurrent cigarette smoking, alcohol use, chronic diseases, snorting, and daytime sleepiness.

Bivariate relationships of sleep duration with the variables of interest are presented in Table 3. Sleep duration was associated with age, race, education, federal poverty level, health insurance, cigarette use, alcohol use, chronic disease, daytime sleepiness, dual use, and electronic cigarette use.

Logistic regression analyses on not having recommended sleep duration for age are presented in Table 4. In the unadjusted logistic regression, participants who reported currently using electronic cigarettes as compared with participants who never used electronic cigarettes had an unadjusted odds ratio of 2.41 (95\% CI: 1.66, 3.50; $P=0.0010)$. Participants who formerly used electronic cigarettes had an unadjusted odds ratio of 1.06 (95\% CI: $0.73,1.53 ; P=0.0861)$. There was an insufficient sample size to conduct an interaction of participants' electronic cigarette use and cigarette use ( 3 electronic cigarette use categories $\times 3$ smoking categories).

In a logistic regression model adjusted with demographic variables (sex, age, race/ethnicity, education, federal poverty level, and insurance), the adjusted odds ratio (AOR) for participants who reported currently using electronic cigarettes as compared with participants who never used electronic cigarettes was 2.41 (95\% CI: 1.66, 3.50; $P=0.0010)$. Participants who formerly used electronic cigarettes had an adjusted odds ratio of 1.09 (95\% CI: $0.76,1.57 ; P=0.1291$ ).

In a second model with the addition of smoking, chronic diseases, and alcohol use, the AOR for participants who reported currently using electronic cigarettes as compared with participants who never used electronic cigarettes was 1.82 (95\% CI: $1.18,2.79 ; P=0.0075)$. Participants who formerly used electronic cigarettes had an adjusted odds ratio of 0.85 (95\% CI: $0.59,1.24 ; P=0.0513$ ).

Sample size limitations precluded further analyses based upon frequency of electronic cigarette subgroup categories of intermediate use, infrequent use, current use, and never use. For dual use logistic regression analyses (presented in Table 5), current dual use was associated with not having recommended sleep duration in the unadjusted $(\mathrm{OR}=2.95(95 \%$ CI: $1.90,4.50 ; P=0.0006)$ ) and both of the adjusted models $(\mathrm{AOR}=2.80(95 \% \mathrm{CI}: 1.68,4.67 ; P=0.0041)$ and $\mathrm{AOR}=$ 2.62 (95\% CI: 1.65, 4.16; $P=0.0010$ ), respectively).

Although not a focus of this study, other factors associated with sleep were also examined. Electronic cigarette use was not associated with snoring $(P=0.4828)$ in bivariate analysis; however, it was associated with snorting $(P=0.0034)$ and daytime sleepiness $(P<0.0001)$. Further analyses with logistic regression failed to reach significance for snorting $\left(\mathrm{OR}_{\mathrm{e} \text {-cigarette use }}=0.58 \quad(95 \% \mathrm{CI}: 0.31,1.07\right.$; $P=0.3200) ; \mathrm{AOR}_{\text {e-cigarette use }}=0.60(0.31,1.15 ; P=0.3822)$; $\mathrm{OR}_{\text {former use }}=0.60 \quad(95 \% \quad \mathrm{CI}: \quad 0.40, \quad 0.89 ; \quad P=0.2649)$; $\left.\mathrm{AOR}_{\text {former use }}=0.60 \quad(0.35,1.02 ; P=0.3135)\right)$. 
TABLE 3: Bivariate analysis of sleep duration with sample variables, NHANES, 2015-2016, $n=2889$.

\begin{tabular}{|c|c|c|c|c|c|}
\hline & \multicolumn{2}{|c|}{$\begin{array}{l}\text { Not recommended sleep duration for } \\
\text { specific age }{ }^{1}\end{array}$} & \multicolumn{2}{|c|}{$\begin{array}{l}\text { May be appropriate sleep duration } \\
\text { for specific age }\end{array}$} & \multirow[t]{2}{*}{$P$ value } \\
\hline & Number & Weighted percent & Number & Weighted percent & \\
\hline Electronic cigarette use & & & & & $<0.0001$ \\
\hline Current & 41 & 13.9 & 150 & 6.3 & \\
\hline Former & 67 & 17.3 & 390 & 18.0 & \\
\hline Never & 276 & 68.8 & 1965 & 75.7 & \\
\hline Cigarette use & & & & & $<0.0001$ \\
\hline Current use & 132 & 35.5 & 494 & 20.1 & \\
\hline Former use & 56 & 16.5 & 424 & 19.8 & \\
\hline Never use & 196 & 48.0 & 1587 & 60.0 & \\
\hline Dual use $\mathrm{e}^{3}$ & & & & & $<0.0001$ \\
\hline Current & Cell size suppressed ${ }^{4}$ & 10.8 & 87 & 3.9 & \\
\hline Former & Cell size suppressed & 2.8 & 85 & 4.3 & \\
\hline Never & Cell size suppressed & 86.4 & 2333 & 91.8 & \\
\hline Sex & & & & & 0.3620 \\
\hline Female & 196 & 48.7 & 1300 & 51.0 & \\
\hline Male & 188 & 51.4 & 1205 & 49.0 & \\
\hline Age in years & & & & & 0.0009 \\
\hline $18-25$ & 48 & 11.0 & 515 & 18.7 & \\
\hline $26-44$ & 152 & 41.3 & 988 & 39.7 & \\
\hline 45-65 & 184 & 47.7 & 1002 & 41.5 & \\
\hline Race/ethnicity & & & & & $<0.0001$ \\
\hline Non-Hispanic white & 82 & 45.2 & 750 & 61.6 & \\
\hline Non-Hispanic black & 138 & 25.7 & 531 & 11.3 & \\
\hline Mexican American & 114 & 19.7 & 790 & 17.0 & \\
\hline Other & 50 & 9.4 & 434 & 10.2 & \\
\hline Education & & & & & $<0.0001$ \\
\hline High school/less & 225 & 57.8 & 1158 & 35.3 & \\
\hline More than high school & 159 & 42.2 & 1347 & 64.7 & \\
\hline Federal poverty level & & & & & $<0.0001$ \\
\hline$\leq 200 \%$ & 251 & 61.1 & 1274 & 37.2 & \\
\hline$>200 \%$ & 133 & 38.9 & 1231 & 62.8 & \\
\hline Health insurance & & & & & 0.0328 \\
\hline Yes & 286 & 78.4 & 1946 & 83.1 & \\
\hline No & 98 & 21.6 & 558 & 16.9 & \\
\hline Body mass index & & & & & 0.0910 \\
\hline Under/normal & 167 & 44.2 & 982 & 39.6 & \\
\hline Over/obese & 209 & 55.8 & 1498 & 60.4 & \\
\hline Alcohol use & & & & & $<0.0001$ \\
\hline No & 116 & 26.9 & 732 & 22.2 & \\
\hline Moderate & 90 & 23.5 & 780 & 38.0 & \\
\hline Heavy & 111 & 33.9 & 609 & 26.5 & \\
\hline Missing & 67 & 15.7 & 384 & 13.4 & \\
\hline Chronic disease $^{1,2}$ & & & & & $<0.0001$ \\
\hline Yes & 176 & 46.8 & 743 & 28.5 & \\
\hline No & 208 & 53.2 & 1762 & 71.5 & \\
\hline
\end{tabular}


TABLE 3: Continued.

\begin{tabular}{|c|c|c|c|c|c|}
\hline & \multicolumn{2}{|c|}{$\begin{array}{l}\text { Not recommended sleep duration for } \\
\text { specific age }\end{array}$} & \multicolumn{2}{|c|}{$\begin{array}{l}\text { May be appropriate sleep duration } \\
\text { for specific age }\end{array}$} & \multirow[t]{2}{*}{$P$ value } \\
\hline & Number & Weighted percent & Number & Weighted percent & \\
\hline Sleepiness & & & & & $<0.0001$ \\
\hline $0-1 /$ month & 122 & 25.8 & 1075 & 39.1 & \\
\hline 2-4/month & 116 & 33.9 & 814 & 34.2 & \\
\hline$\geq 5 /$ month & 146 & 40.3 & 616 & 26.7 & \\
\hline
\end{tabular}

Abbreviations: wt: weighted; HS: high school. ${ }^{1}$ Based upon National Sleep Foundation (https://www.sleepfoundation.org/press-releasenational-sleepfoundation-recommends-new-sleep-times). ${ }^{2}$ Chronic disease = positive response to having arthritis, cardiovascular disease, depression, emphysema, or diabetes. ${ }^{3}$ Dual use current $=$ smoking cigarettes and using electronic cigarettes over the previous 30 days; former dual use $=$ reporting having smoked $>100$ lifetime cigarettes and having ever used electronic cigarettes; never = reporting never having smoked $>100$ cigarettes and reporting never having tried electronic cigarettes. ${ }^{4}$ Numerical entries into the cell categories are suppressed for participant confidentiality.

In unadjusted logistic regression, e-cigarette use was more likely to be associated with daytime sleepiness $(\mathrm{OR}=0.36(0.26,0.52 ; P=0.0008))$ and former e-cigarette use was not $(P=0.9219)$. Further adjusted analyses were not reliable due to the lack of degrees of freedom in computing the results; however, with degrees of freedom set at infinity, daytime sleepiness failed to reach significance $(P=0.3845)$. These results are not presented in a tabular format.

\section{Discussion}

In this study, the researchers report that participants who currently use electronic cigarettes were more likely to have less sleep as compared with participants who never used electronic cigarettes. After adjusting for sociodemographic variables and variables from the Unifying Energy Allocation Model of Sleep, current electronic cigarette use remained associated with higher odds of less sleep duration. As mentioned earlier, the wake-sleep predominant functions are altered by many factors. Our results suggest that electronic cigarette smoking is one such factors; however, as a crosssectional study, causality cannot be definitively determined by this study: it is possible that electronic cigarette use does impact sleep duration, and it is also possible that when there is reduced sleep duration, there is a greater likelihood of using electronic cigarettes.

In an online search for electronic cigarette use and harm, the first thirty results indicated warnings of dangers associated with electronic cigarettes; further searches described electronic cigarettes as less harmful than smoking, as having a role in tobacco harm reduction. News reports of 2561 hospitalizations and 55 deaths due to lung injuries from electronic cigarettes [29] have increased awareness of the potential dangers of electronic cigarettes.

In support of the potential causal effect, many e-fluids contain stimulants (such as nicotine) that are known to impact sleep. However, there are several electronic cigarette fluids that do not have nicotine in the e-fluids. An alternate possible mechanism for causality is through the link that researchers found between e-liquids with vitamin $\mathrm{E}$ acetate and adverse respiratory outcomes. Coughing, airway irritation, and chest pain were associated with electronic cigarette use $[29,30]$, and such factors may interrupt sleep duration. Although vitamin E acetate seems to be a significant airway irritant, there are many different substances and product sources that are yet to be investigated [29].

Data are scant on electronic cigarette use behavior. Therefore, novel approaches for research on electronic cigarette use behavior are needed. For example, researchers are using genetic analyses to examine tobacco use and other substance use behavior [31]. In a study using Mendelian randomization to calculate genetic correlations and causal effects, researchers found novel genetic correlations between smoking (initiation and cigarettes per day) and reduced sleep behavior [32]. Since the development of the genome-wide association study and the Sequencing Consortium of Alcohol and Nicotine Use, hundreds of novel loci, specifically 564 independent genetic variants assigned to 405 genes, have been discovered to be associated with smoking and drinking behaviors [32]. Genetic analyses between electronic cigarette use and behavioral outcomes may provide similar information concerning genes associated with electronic cigarette use behavior. Unhealthy substance use behavior among adolescents, including tobacco use behavior, declined over the last decade. However, in 2017, approximately 3.6 million U.S. middle school and high school students were using some form of tobacco, selecting electronic vapor products as the most popular product [33]. It is imperative to understand the mechanisms leading to electronic cigarette use.

4.1. Strengths and Limitations. The strengths of this study include the national representative sample, the standardized data collection approach, and the ability to adjust for many variables. Though this study has many strengths, there are also certain limitations. NHANES data are observational, and causal inferences cannot be drawn between sleep duration and electronic cigarette use.

The data for sleep duration and electronic cigarette use were self-reported and not objectively measured. The data may be subjected to recall bias. Although sleep can be measured on several parameters, such as sleep time duration, sleep efficiency, sleep onset latency, and the number of awakenings, the researchers considered only sleep time duration in this study. It is possible that the use of a single measure may not fully capture the clinical construct of sleep quality.

Electronic cigarette use was defined as current use (using electronic cigarettes within the previous 30 days), former use (ever having used electronic cigarettes), and never use. This is 


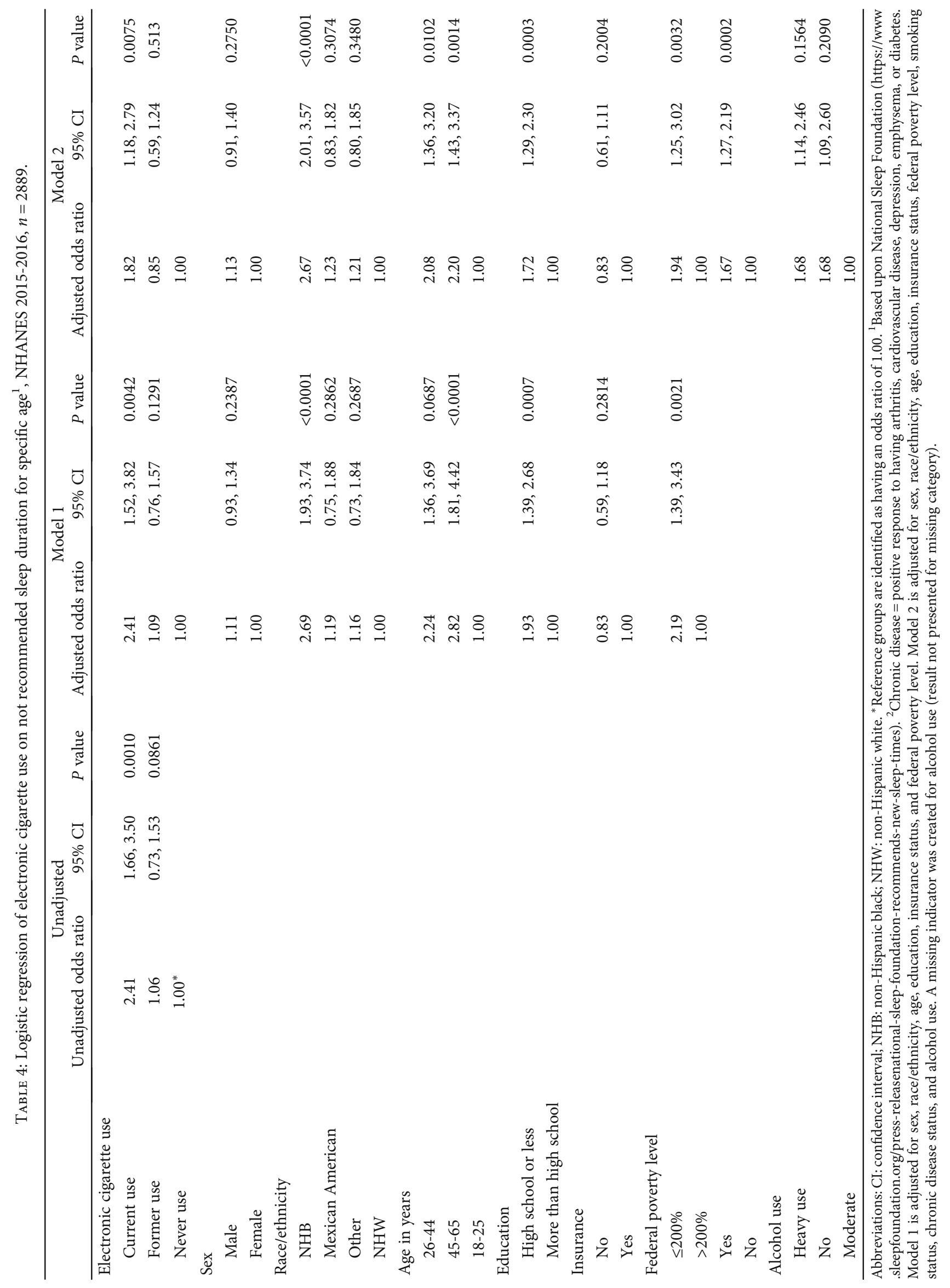




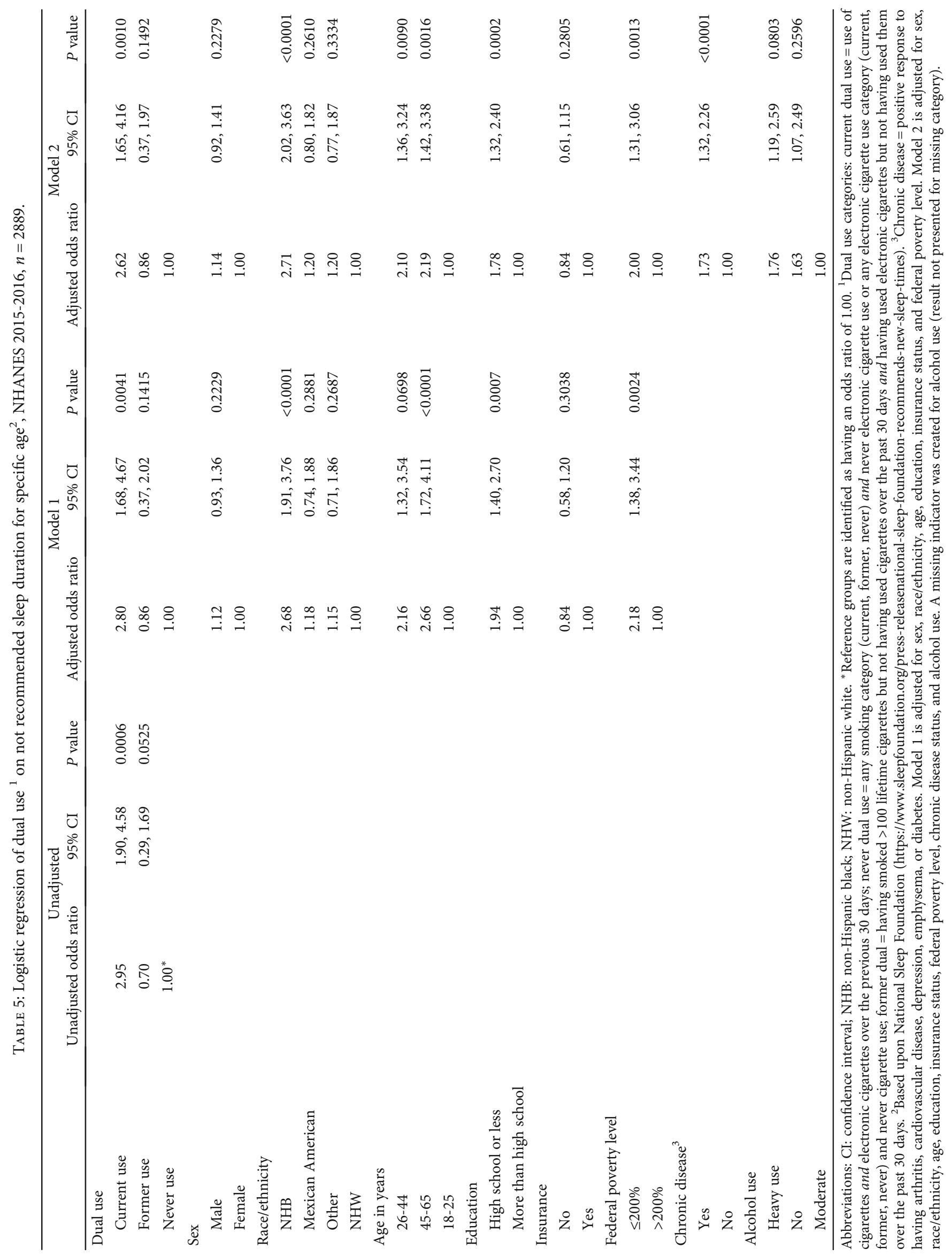


the conventional method of describing cigarette use; however, the patterns of electronic cigarette use are different from cigarette use and are not standardized for research. Electronic cigarette use is complex and often involves dual use [26-28]. The synergistic effect of electronic cigarettes and conventional tobacco use (dual use) can be much greater than using only one inhalant [34]. The authors provided additional analyses on dual use to address this limitation.

\section{Conclusion}

Participants who currently use electronic cigarettes were 1.82 times more likely not to get their recommended sleep time duration as compared with participants who never used electronic cigarettes. With sleep time duration being a major factor for proper body function and repair, this study can serve as confirmation that the use of electronic cigarettes is not a harmless health behavior.

\section{Data Availability}

The data used to support the findings of this study are available from the corresponding author upon request.

\section{Disclosure}

The content is solely the responsibility of the authors and does not necessarily represent the official views of the National Institutes of Health. The funders had no role in study design, data collection and analysis, decision to publish, or preparation of the manuscript.

\section{Conflicts of Interest}

The authors declare that they have no conflicts of interest.

\section{Acknowledgments}

Study data were collected and managed using REDCap electronic data capture tools hosted at West Virginia University. The research reported in this publication was supported by the National Institute of General Medical Sciences of the National Institutes of Health under Award Number U54GM104942.

\section{References}

[1] T. Z. Liu, C. Xu, M. Rota et al., "Sleep duration and risk of allcause mortality: a flexible, non-linear, meta-regression of 40 prospective cohort studies," Sleep Medicine Reviews, vol. 32, pp. 28-36, 2017.

[2] Y. Li, S. Cai, Y. Ling et al., "Association between total sleep time and all cancer mortality: non-linear dose-response meta-analysis of cohort studies," Sleep Medicine, vol. 60, pp. 211-218, 2019.

[3] O. Itani, M. Jike, N. Watanabe, and Y. Kaneita, "Short sleep duration and health outcomes: a systematic review, metaanalysis, and meta-regression," Sleep Medicine, vol. 32, pp. 246-256, 2017.

[4] S. Cohrs, A. Rodenbeck, D. Riemann et al., "Impaired sleep quality and sleep duration in smokers-results from the
German Multicenter Study on Nicotine Dependence," Addiction Biology, vol. 19, no. 3, pp. 486-496, 2014.

[5] E. N. Dugas, M. P. Sylvestre, E. K. O'Loughlin et al., "Nicotine dependence and sleep quality in young adults," Addictive Behaviors, vol. 65, pp. 154-160, 2017.

[6] A. C. Hayley, C. Stough, and L. A. Downey, "Dsm-5 tobacco use disorder and sleep disturbance: findings from the National Epidemiologic Survey on Alcohol and Related Conditions-III (NESARC-III)," Substance Use \& Misuse, vol. 52, no. 14, pp. 1859-1870, 2017.

[7] G. J. Lavigne, F. Lobbezoo, P. H. Rompré, T. A. Nielsen, and J. Montplaisir, "Cigarette smoking as a risk factor or an exacerbating factor for restless legs syndrome and sleep bruxism," Sleep, vol. 20, no. 4, pp. 290-293, 1997.

[8] D. W. Wetter, T. B. Young, T. R. Bidwell, M. S. Badr, and M. Palta, "Smoking as a risk factor for sleep-disordered breathing," Archives of Internal Medicine, vol. 154, no. 19, pp. 22192224, 1994.

[9] L. Zhang, J. Samet, B. Caffo, and N. M. Punjabi, "Cigarette smoking and nocturnal sleep architecture," American Journal of Epidemiology, vol. 164, no. 6, pp. 529-537, 2006.

[10] J. O. Tweed, S. H. Hsia, K. Lutfy, and T. C. Friedman, "The endocrine effects of nicotine and cigarette smoke," Trends in Endocrinology and Metabolism, vol. 23, no. 7, pp. 334-342, 2012.

[11] H. L. Mathews and J. A. Stitzel, "The effects of oral nicotine administration and abstinence on sleep in male C57BL/6J mice," Psychopharmacology, vol. 236, no. 4, pp. 1335-1347, 2019.

[12] A. Jaehne, T. Unbehaun, B. Feige et al., "Sleep changes in smokers before, during and 3 months after nicotine withdrawal," Addiction Biology, vol. 20, no. 4, pp. 747-755, 2015.

[13] A. Cohen, N. Ben Abu, and I. Haimov, "The interplay between tobacco dependence and sleep quality among young adults," Behavioral Sleep Medicine, vol. 18, no. 2, pp. 163-176, 2020.

[14] Y. Varol, C. Anar, O. E. Tuzel, S. Z. Guclu, and Z. Z. Ucar, "The impact of active and former smoking on the severity of obstructive sleep apnea," Sleep and Breathing, vol. 19, no. 4, pp. 1279-1284, 2015.

[15] C. Dinaker and G. T. O'Connor, "The health effects of electronic cigarettes," NEJM, vol. 375, no. 14, pp. 1372-1381, 2016.

[16] S. H. Zhu, J. Y. Sun, E. Bonnevie et al., "Four hundred and sixty brands of e-cigarettes and counting: implications for product regulation," Tobacco Control, vol. 23, Supplement 3, pp. iii3iii9, 2014.

[17] T. Basáñez, A. Majmundar, T. B. Cruz, J. P. Allem, and J. B. Unger, "E-cigarettes are being marketed as "vitamin delivery" devices," American Journal of Public Health, vol. 109, no. 2, pp. 194-196, 2019.

[18] C. A. Schoenborn and R. M. Gindi, Electronic Cigarette Use among Adults: United States, US Department of Health and Human Services, Centers for Disease Control and Prevention, National Center for Health Statistics, Hyattsville, MD, USA, 2014, Published in NCHS data brief October, 2015.

[19] Centers for Disease Control and Prevention, About Electronic Cigarettes (E-Cigarettes), Office on Smoking and Health, National Center for Chronic Disease Prevention and Health Promotion, 2018, https://www.cdc.gov/tobacco/basic_ information/e-cigarettes/about-e-cigarettes.html.

[20] K. M. Berry, J. L. Fetterman, E. J. Benjamin et al., “Association of electronic cigarette use with subsequent initiation of tobacco 
cigarettes in US youths," JAMA Network Open, vol. 2, no. 2, article e187794, 2019.

[21] S. Boddu, C. M. Bojanowski, E. Scholten et al., "Investigating the effects of different forms of tobacco use on sleep quality and cough: a matter of gender? American Thoracic Society," American Journal of Respiratory and Critical Care Medicine, vol. 199, article A1180, 2019.

[22] M. S. Dunbar, J. S. Tucker, B. A. Ewing et al., "Frequency of E-cigarette use, health status, and risk and protective health behaviors in adolescents," Journal of Addiction Medicine, vol. 11, no. 1, pp. 55-62, 2017.

[23] M. H. Schmidt, "The energy allocation function of sleep: a unifying theory of sleep, torpor, and continuous wakefulness," Neuroscience \& Biobehavioral Reviews, vol. 47, pp. 122-153, 2014.

[24] NHANES, 2015-2016, "National Health and Examination Survey, 2015-2016 Questionnaires, Datasets, and Related Documentation," Centers for Disease Control and Prevention. National Center for Health Statistics, 2018, https://wwwn.cdc .gov/nchs/nhanes/Continuous Nhanes/Default .aspx?BeginYear=2015.

[25] National Sleep Foundation, National Sleep Foundation recommends new sleep times. News Release, Washington, DC, USA, 2015, https://www.sleepfoundation.org/press-releasenationalsleep-foundation-recommends-new-sleep-times.

[26] M. S. Amato, R. G. Boyle, and D. Levy, "How to define e-cigarette prevalence? Finding clues in the use frequency distribution," Tobacco Control, vol. 25, no. e1, pp. e24-e29, 2016.

[27] A. C. Villanti, J. L. Pearson, A. M. Glasser et al., "Frequency of youth E-cigarette and tobacco use patterns in the U.S.: measurement precision is critical to inform public health," Nicotine \& Tobacco Research, vol. 19, pp. 1345-1350, 2017.

[28] J. L. Pearson, S. C. Hitchman, L. S. Brose et al., "Recommended core items to assess e-cigarette use in population-based surveys," Tobacco Control, vol. 27, no. 3, pp. 341-346, 2018.

[29] Centers for Disease Control and Prevention, Outbreak of lung injury associated with the use of E-cigarette, or vaping products, Office on Smoking and Health, National Center for Chronic Disease Prevention and Health Promotion, 2019, https:// www.cdc.gov/tobacco/basic_information/e-cigarettes/severelung-disease.html.

[30] J. B. Wang, J. E. Olgin, G. Nah et al., "Cigarette and e-cigarette dual use and risk of cardiopulmonary symptoms in the Health eHeart Study," PLoS One, vol. 13, no. 7, article e0198681, 2018.

[31] A. G. Allegrini, K. J. H. Verweij, A. Abdellaoui et al., "Genetic vulnerability for smoking and cannabis use: associations with e-cigarette and water pipe use," Nicotine \& Tobacco Research, vol. 21, no. 6, pp. 723-730, 2019.

[32] A. W. Bergen, E. K. Do, L. S. Chen, and S. P. David, "Tobacco genomics: complexity and Translational challenges," Nicotine \& Tobacco Research, vol. 21, no. 6, pp. 705-706, 2019.

[33] Centers for Disease Control and Prevention (b), "QuickStats: cigarette smoking status among current adult E-cigarette users, by age group-National Health Interview Survey, United States, 2015," MMWR, vol. 65, article 1177, 2016.

[34] S. A. Boddu, C. M. Bojanowski, M. T. Lam et al., "Use of Electronic Cigarettes with conventional tobacco is associated with decreased sleep quality in women," American Journal of Respiratory and Critical Care Medicine, vol. 200, no. 11, pp. 1431-1434, 2019. 